Preprint typeset in JHEP style - HYPER VERSION

LU-TP 02-34

\title{
Hadronic Collisions in the Linked Dipole Chain Model
}

\author{
Gösta Gustafson, Leif Lönnblad and Gabriela Miu \\ Dept. of Theoretical Physics, Sölvegatan 14A, S-223 62 Lund, Sweden \\ E-mail: Gosta.Gustafson@thep.lu.se, Eeif.Lonnblad@thep.lu.se and Gabriela.Miu@thep.lu.se
}

\begin{abstract}
We apply the Linked Dipole Chain model to hadronic collisions using a modified version of the LDCMC Monte Carlo program. In particular we investigate the effects of multiple scatterings, which in this framework are reformulated in terms of the production of multiple chains of dipoles. We find, among other things, that this way of describing multiple scatterings is less sensitive to the treatment of soft, non-perturbative, scatterings. Although the results presented here are only at parton level, by comparing with corresponding results from PYTHIA, we are confident that our model will be able to describe essential features of high energy hadronic collisions, including underlying events and jetpedestal effects in high $p_{\perp}$ events. The model should also be applicable to estimates of transverse energy flow in high energy heavy ion collisions, of interest in analyses of signals for quark-gluon plasma formation.
\end{abstract}

Keywords: QCD, Jets, Parton Model, Phenomenological Models, Hadronic Colliders. 


\section{Contents}

1. Introduction 1

2. Small- $x$ physics in DIS

2.1 CCFM Evolution 1

2.2 The Linked Dipole Chain Model 5

3. The Linked Dipole Chain Model for Hadronic Collisions 7

3.1 Hard Chains 6

3.2 Soft Chains 8

3.3 Final State Bremsstrahlung 8

4. Multiple scatterings in hadronic collisions 9

4.1 Impact-Parameter Dependence 10

5. Monte Carlo implementation 11

5.1 Colour Connections

5.2 Energy-Momentum Conservation 13

6. Results 13

6.1 Fitting procedure 14

6.2 Chain multiplicity 15

6.3 Inclusive jet production 17

6.4 Minijet distributions 18

6.5 The Pedestal Effect and Underlying events 19

6.6 Cutoff Dependence 20

7. Conclusions 21

\section{Introduction}

Although the main aim for the future Large Hadron Collider at CERN is to discover and study the Higgs boson, and to possibly discover new physics, it is important to keep in mind that our understanding of the strong interactions, which make up the bulk of the cross section in hadronic collisions, is far from complete. The hard production of large transverse momentum jets may be described to a reasonable precision by perturbative QCD, but for the dominating soft and semi-hard interactions we have to rely on phenomenological models. There may be some gold-plated signals for new physics, where these so-called minimum bias interactions may be disregarded, but as soon as observables include jets, a 
more detailed understanding of the event structure is needed. Not only is the luminosity so high at the LHC that any signal event will be accompanied by several overlayed minimum bias collisions. In addition the inclusive cross section for parton-parton scatterings becomes larger than the total non-diffractive cross section already at present day energies, which implies that there are several parton-parton scatterings in each single hadron-hadron collision. Such additional scatterings can be rather soft, and can therefore not be described by purely perturbative calculations. The production of minijets is also of interest in high energy heavy ion collisions, and the analysis of signals for plasma formation. They are essential for the initial conditions in studies of the flow in either a quark-gluon plasma or a hadronic phase [1].

Further studies of hadronic collisions are also interesting in its own right, in order to understand the nature of strong interaction and the interplay between soft and hard physics. Many models have been presented to describe and simulate hadronic collisions. The Fritiof model [2] successfully describes hadron collisions at lower energies, up to the ISR range. It is based on a soft momentum transfer and gluon bremsstrahlung due to the separation of colour charges. These charges are in the model connected by strings of the same nature as in $e^{+} e^{-}$-annihilation. For higher energies hard sub-collisions were added [3]. The formalism did, however, not give a unique description for how to combine the soft and hard subprocesses, and therefore the predictive power at high energies is reduced.

The Dual Parton Model (DPM) [4, 5] aims at a combined description of soft and hard interactions in an eikonalized form, including besides a soft and a hard pomeron also tripleand loop-pomeron contributions. The aim is to describe not only non-diffractive events, but also elastic scattering and single and double diffraction, and the model is able to reproduce a large set of experimental data [6].

At high energies, in the $\bar{p} p$ collider regime, the cross section for hard or semi-hard parton sub-collisions becomes large, and the properties of non-diffractive events appear to be dominated by these subprocesses. In this energy range it is therefore essential to have a good description of jets from hard parton collisions, including initial and final state bremsstrahlung. In ISAJET [7] and HERWIG [8] a description of the hard sub-collisions is complemented by an "underlying event" due to interactions between the beam remnants. In PYтніA [9] this underlying event is modeled by including multiple parton interactions in the generation of the hard parton collision, rather than adding them as an independent component to the hard sub-collisions.

Even if the parton-parton collisions are of perturbative nature, with a $p_{\perp}$ above a couple of $\mathrm{GeV}$, there are many difficulties, which cannot be solved by perturbative methods alone. The parton sub-collisions are typically modeled using collinear factorization. This gives a divergent expression for the inclusive cross section at small $p_{\perp}$, which has to be treated by some kind of cutoff. Clearly collisions with large impact parameter must be suppressed due to screening effects, when the impact parameter is larger than the proton radius. The impact parameter dependence is also expected to influence the correlations between sub-collisions in a single event. It is natural to assume that central collisions have a higher activity than more peripheral collisions and therefore, on average, a larger number of sub-collisions [10]. Both these effects, the $p_{\perp}$ cutoff and the multiplicity correlations, 
have to be adjusted to experimental data, which makes it more difficult to make reliable extrapolations to higher energies.

In collinear factorization the hard partonic cross section is convoluted with parton densities, which are evolved using DGLAP [11, 12, 13, 14] evolution. The parton densities are fitted to data on e.g. $F_{2}$ in DIS and high $E_{\perp}$ jets in hadronic collisions. For moderate $p_{\perp}$ at very large c.m. energies, $\sqrt{s}$, the parton evolution contains large logarithms of $s / p_{\perp}^{2}$ which need to be resummed to all orders. For these $p_{\perp}$ values it would therefore be more appropriate to use BFKL evolution [15, 16], which gives the correct description, at least for asymptotic energies and fixed coupling. The BFKL evolution is related to $k_{\perp}$-factorization, and in ref. [17] it is argued that this approach gives a dynamical suppression for small $p_{\perp}$, which reduces the infrared sensitivity. This is mainly a consequence of a suppression of the partonic cross section when the virtualities of the colliding partons are larger than the transverse momentum exchanged in the hard collision.

The study in ref. [17] is based on the Linked Dipole Chain (LDC) model [18, 19] for DIS. This model is based on the observation that the dominant features of the parton evolution is determined by a subset of emitted gluons, which are ordered in both positive and negative light-cone components. These primary gluons make up a linked chain of colour dipoles, and the sum over all such possible chains, convoluted with non-perturbative input parton densities, will give the proton structure function. (For exclusive properties of the final states also final state radiation has to be included.) The LDC model is completely forward-backward symmetric - i.e. it does not matter if the evolution is performed from the proton towards the virtual photon or vice versa. This means that LDC is also very suited for a description of hadronic collisions, where the sum over all possible chains, convoluted with input parton densities from both hadrons, will give the inclusive partonic cross section. The fact that transverse momenta can go up and down in a single parton chain implies that such a chain can correspond to more than one hard parton-parton sub-collision. Thus one type of correlations between the sub-collisions is automatically included in the LDC formalism. However, even so the per chain cross section exceeds the total non-diffractive cross section. Therefore there will in general be several dipole chains stretched between two colliding hadrons, and it is also in this approach necessary to make assumptions about the impact parameter dependence, now for the distribution in the number of chains in one event.

In this paper we will investigate the effects of such multiple chains in more detail, using the LDC event generator LDCMC [20], modified to generate hadronic collisions. The investigation will still be on a somewhat qualitative level since effects of colour connection between the chains, and of hadronization are not included. Therefore we will here compare our results with the PYTHIA generator on parton level rather than with experimental data.

A very essential result from these MC studies is the fact that the number of chains per event is stable against variations of the $p_{\perp}$ cutoff in the perturbative parton evolution. When fitting experimental data on $F_{2}$, the input soft gluon distribution is adjusted, and corresponds to a scale given by the cutoff. The soft input gluons can also form direct chains between the colliding hadrons, and these chains are also allowed to emit final state bremsstrahlung below the cutoff. If the soft cutoff is increased there will be fewer hard 
chains, which is compensated by a more singular input gluon distribution. As we will show below, when the cutoff is increased, the decrease in the number of hard chains is compensated by a larger number of soft chains (which are now also allowed to radiate more). Thus the total number of chains in $p p$ collisions can be fully determined by data on $F_{2}$, without any additional adjustable free parameter.

The outline of this paper is as follows. In section 2 we give some further comments on parton evolution in DIS, including a brief introduction to the LDC model. In section 3 we discuss the application of the LDC model for hadronic collisions. Then in section 4 we discuss some general aspects of multiple scatterings, in particular how they are included in the PyTHIA generator and how we treat them in the present analysis. The technical details of the Monte-Carlo implementation are discussed in section 5 . In section 6 we present some preliminary results, followed by conclusions in section 7 .

\section{Small- $x$ physics in DIS}

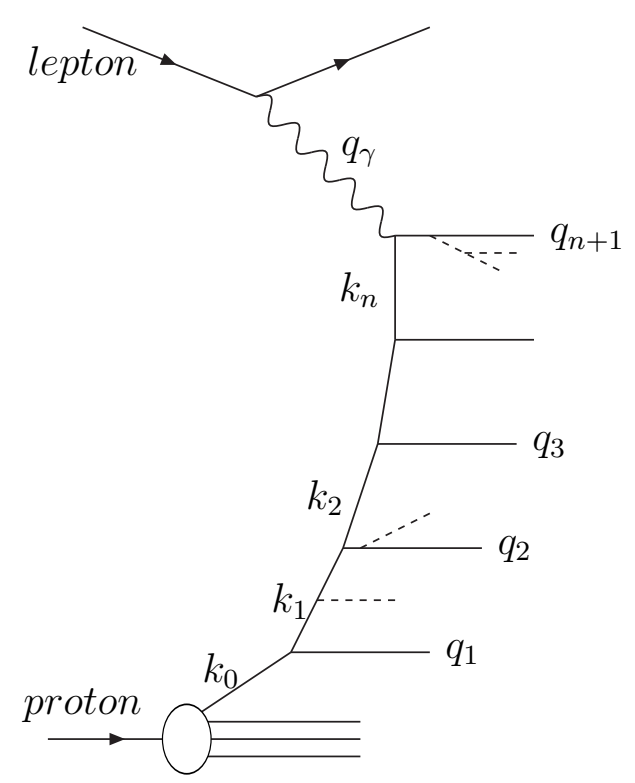

Figure 1: A fan diagram for a DIS event. The quasi-real partons from the initial-state radiation are denoted $q_{i}$, and the virtual propagators $k_{i}$. The dashed lines denote final-state radiation.
Effects of BFKL evolution have been studied extensively at HERA, where BFKL predicted a strong power-like increase of $F_{2}$ for small $x$. It turns out, however, that BFKL suffers from huge next-to-leading order corrections [21] and it has been very difficult to obtain reliable predictions, especially when it comes to non-inclusive observables involving final-state hadrons. More reliable predictions for final-state observables can be obtained using CCFM evolution [22, 23], which agrees with BFKL in the asymptotic limit, but also approximates DGLAP for large $x$. The central feature of the CCFM model is the angular ordering of the parton chains contributing to the proton structure function, which is a consequence of colour coherence or soft gluon interaction. The parton chains are ordered in energy (or positive light-cone momentum $q_{+}$) and in angle (or rapidity $\left.y=\frac{1}{2} \ln \left(q_{+} / q_{-}\right)\right)$. (We use $q_{i}$ and $k_{i}$ to denote the real emitted partons and the virtual links respectively, as indicated in figure 11.) Other kinematically allowed emissions (symbolized by the dashed

lines in figure 11) are treated as final-state emissions.

\subsection{CCFM Evolution}

Like BFKL evolution, the CCFM model is based on the $k_{\perp}$-factorization formalism, and 
the unintegrated distribution function is given by

$$
\begin{aligned}
\mathcal{G}\left(x, k_{\perp}^{2}, \bar{q}\right) \sim & \sum_{n} \int \prod^{n} \bar{\alpha} d z_{i} \frac{d^{2} q_{\perp i}}{\pi q_{\perp i}^{2}}\left(\frac{1}{z_{i}} \Delta_{n e}\left(z_{i}, k_{\perp i}^{2}, \bar{q}_{i}\right)+\frac{1}{1-z_{i}}\right) \Delta_{S} \times \\
& \delta\left(x-\Pi z_{i}\right) \theta\left(\bar{q}_{i}-\bar{q}_{i-1} z_{i-1}\right) \delta\left(k_{\perp}^{2}-k_{\perp n}^{2}\right) \theta\left(\bar{q}-\bar{q}_{n} z_{n}\right)
\end{aligned}
$$

where the non-eikonal and the Sudakov form factors are given by

$$
\Delta_{n e}=\exp \left(-\bar{\alpha} \ln \frac{1}{z} \ln \frac{k_{\perp}^{2}}{z \bar{q}^{2}}\right) ; \quad \Delta_{S}=\exp \left(-\bar{\alpha} \int \frac{d q_{\perp i}^{2}}{q_{\perp i}^{2}} \frac{d z}{1-z} \Theta_{\text {order }}\right)
$$

Here $\bar{\alpha} \equiv 3 \alpha_{s} / \pi$, the splitting parameter $z$ is defined as $z_{i}=k_{+, i} / k_{+, i-1}$, and the kinematic range allowed by the ordering constraints is specified by $\Theta_{\text {order }}$. The quantity $\bar{q}_{i}$ is defined by $\bar{q}_{i} \equiv q_{\perp i} /\left(1-z_{i}\right)$, which implies that the angular ordering condition is satisfied by the constraint $\bar{q}_{i}>\bar{q}_{i-1} z_{i-1}$, accounted for by a $\theta$-function in eq. (2.1). The CCFM model has been developed assuming purely gluonic chains, and only the singular terms in the splitting functions, proportional to $1 / z$ and $1 /(1-z)$, are included in a fully consistent way. The $1 / z$ pole is most important in the BFKL region for small $x$, while the $1 /(1-z)$ pole and the Sudakov form factor are essential for larger $x$ and large $k_{\perp}$. We also note that the distribution function, $\mathcal{G}$, depends on two separate scales. Besides the transverse momentum, $k_{\perp}$, of the interacting gluon, it also depends on $\bar{q}$, which determines an angle beyond which there is no (quasi-) real parton in the chain.

A Monte Carlo implementation of CCFM, CASCADE [24], reproduces a wide range of final-state observables at HERA, although there are still some unsolved questions. Since CCFM does not include the non-singular terms in the gluon splitting function, DGLAP is not fully reproduced in the relevant limit, and including non-singular terms degrades the reproduction of HERA data, especially for observables which are supposed to be sensitive to BFKL effects, such as forward jet cross sections [25].

\subsection{The Linked Dipole Chain Model}

The Linked Dipole Chain (LDC) model [18, 19] is a reformulation and generalization of CCFM. It is based on the observation that the dominant features of the parton evolution is determined by a subset of emitted gluons, which are ordered in both positive and negative light-cone components, and also satisfies the relation

$$
q_{\perp i}>\min \left(k_{\perp i}, k_{\perp, i-1}\right) .
$$

In LDC this subset of "primary" gluons forms a chain of initial state bremsstrahlung (ISB), and all other emissions are treated as final state bremsstrahlung (FSB).

This redefinition of the ISB-FSB separation implies that one single chain in the LDC model corresponds to a set of CCFM chains. As was shown in ref. 18], when one considers the contributions from all chains in this set, with their corresponding non-eikonal form factors, they just add up to one. Thus, the non-eikonal form factors do not appear explicitly 
in LDC, and the gluon distribution function is given by

$$
\begin{aligned}
\mathcal{G}\left(x, k_{\perp}^{2}\right) \sim & \sum_{n} \int \prod^{n} \bar{\alpha} d z_{i} \frac{d^{2} q_{\perp i}}{\pi q_{\perp i}^{2}} P\left(z_{i}\right) \Delta_{S} \times \\
& \theta\left(q_{+, i-1}-q_{+i}\right) \theta\left(q_{-i}-q_{-, i-1}\right) \delta\left(x-\Pi z_{i}\right) \delta\left(\ln k_{\perp}^{2}-\ln k_{\perp n}^{2}\right) .
\end{aligned}
$$

Here $P(z)$ is the full gluonic splitting function, and $\Delta_{S}$ the associated Sudakov form factor, defined by

$$
\Delta_{S}=\exp \left(-\bar{\alpha} \int \frac{d q_{\perp i}^{2}}{q_{\perp i}^{2}} z d z P(z) \Theta_{\text {order }}\right)
$$

where again $\Theta_{\text {order }}$ specifies the region of phase space allowed by the ordering constraints. The result of this reformulation is a simpler form for the unintegrated distribution functions, which essentially depends only on a single scale, $k_{\perp}^{2}$. As many of the gluons which make up the initial-radiation chain in the CCFM model are treated as final state radiation in the LDC formalism, most of the problem of angular ordering is postponed to the treatment of the final state radiation. The ordering of the LDC evolution in both $q_{+}$and $q_{-}$, and the fact that the $1 / z$ pole in the splitting function is here not associated with an non-eikonal form factor, imply that this contribution is completely forward-backward symmetric. Therefore the chain in figure 1 can be thought of either as an evolution from the proton towards the photon or, equivalently, from the photon towards the proton end. The $1 /(1-z)$ pole in $P(z)$ and the Sudakov form factor are most essential in the DGLAP region, where chains with ordered $q_{\perp}$ values dominate and large $z$-values are important. In the LDC model the expression in eq. (2.4) is symmetrized so that steps downwards in transverse momentum with large values of the backwards splitting parameter $z_{-i}=k_{-i} / k_{-, i+1}$ are weighted by the splitting function $P\left(z_{-i}\right)$ and a correspondingly defined Sudakov form factor. The absence of the non-eikonal form factor also implies that quark links and non-singular terms in the splitting function can be included in a natural and straightforward way.

The set of "primary" gluons in eq. 2.4) make up a chain of linked colour dipoles, and the sum over all such possible chains, convoluted with non-perturbative input parton densities, will give the proton structure function. Besides this inclusive description of the events, the result in eq. (2.4) can also be interpreted as the production probability for an exclusive final state. We note, however, that due to the different separation between ISB and FSB in LDC and CCFM or other approaches, it is important to include final state emissions before comparing results for exclusive final states with results from other formalisms or with experimental data.

The LDC model is also implemented in a MC event generator, LDCMC. When the soft input parton densities are adjusted to fit data on $F_{2}$, the model is able to reproduce a wide range of HERA data [20]. The resulting integrated gluon distribution also agrees well with global fits by the CTEQ [26] and MRST [27] collaborations [28]. However, as for the CCFM Monte Carlos, the data for forward jets can only be described if non-singular terms are omitted from the gluon splitting function.

The fact that the LDC formalism is manifestly forward-backward symmetric implies that it automatically takes into account contributions from "resolved virtual photons", 


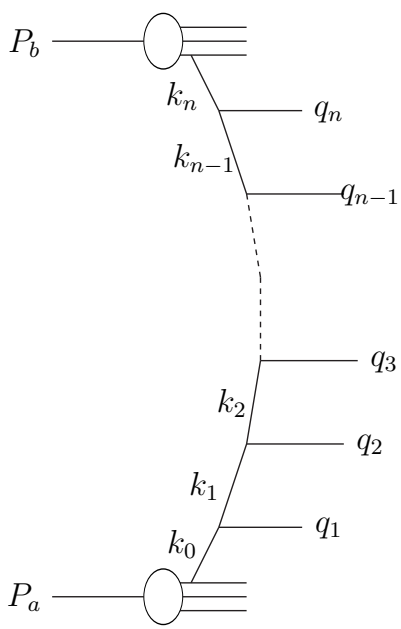

(a)

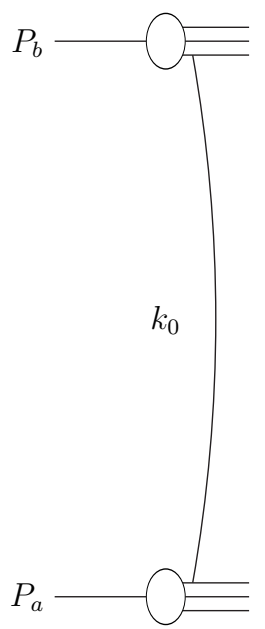

(b)

Figure 2: (a) A chain of $n$ initial state emissions in a hadronic collision. (b) An exchange of a single soft gluon without initial state emissions.

where the chain describes evolution from the proton and photon ends towards a central hard parton sub-collision. This property is a consequence of the specific choice of separation between initial and final state emissions adopted in the LDC model, and it means that the formalism is also very well suited to describe hard interaction in hadron-hadron collisions, as will be discussed in more detail in the following section.

\section{The Linked Dipole Chain Model for Hadronic Collisions}

As mentioned in section 2, to get the gluon distribution function in a proton, the perturbative chains in eq. (2.4) have to be convoluted with non-perturbative input parton densities, $g_{0}\left(x_{0}, k_{\perp 0}^{2}\right)$, for $k_{\perp}^{2}=k_{\perp 0}^{2}$ :

$$
g\left(x, k_{\perp}^{2}\right)=\int d x_{0} d x^{\prime} \mathcal{G}\left(x^{\prime}, k_{\perp}^{2} ; k_{\perp 0}^{2}\right) g_{0}\left(x_{0}, k_{\perp 0}^{2}\right) \delta\left(x_{0} x^{\prime}-x\right)
$$

As we are assuming a running coupling, which diverges for small $q_{\perp}$, it is necessary to introduce a cutoff, $k_{\perp 0}$, for the $q_{\perp i}$ distributions in eq. (2.4) (see e.g. [19]). In eq. (3.1) this is explicitely indicated as an argument in $\mathcal{G}$. Thus $g_{0}\left(x_{0}, k_{\perp 0}^{2}\right)$ is assumed to describe all chains with transverse momenta below the cutoff $k_{\perp 0}$.

\subsection{Hard Chains}

The fact that $\mathcal{G}$ is completely forward-backward symmetric, as discussed in section 2, now implies that the cross section, $\sigma_{c}$, for formation of a chain between two colliding protons is directly obtained if the distribution in eq. (3.1) is convoluted with a parton distribution from the upper proton in figure 2. Thus we find:

$$
\sigma_{c}=\frac{\pi^{3}}{2 k_{\perp 0}^{2}} \int d x_{0+} d x d x_{0-} g_{0}\left(x_{0+}, k_{\perp 0}^{2}\right) \mathcal{G}\left(x, k_{\perp 0}^{2} ; k_{\perp 0}^{2}\right) g_{0}\left(x_{0-}, k_{\perp 0}^{2}\right) \delta\left(x_{0+} x x_{0-}-k_{\perp 0}^{2} / s\right),
$$


with $x_{0+}=k_{0+} / P_{a+}$ and $x_{0-}=k_{n-} / P_{b-}$. The normalization factor $\pi^{3} / 2$ originates from the fact that a hard scattering in the middle of the chain, with $q_{\perp i}^{2} \approx k_{\perp i}^{2} \approx q_{\perp, i+1}^{2}$, is in eq. (2.4) given a weight

$$
\frac{\bar{\alpha}^{2}}{q_{\perp i}^{2} q_{\perp, i+1}^{2}} \approx \frac{\bar{\alpha}^{2}}{\hat{t}^{2}}
$$

where $\hat{t} \approx-k_{\perp i}^{2}$. This should be compared with the well-known cross section for gluongluon scattering, which for $\hat{s}>>|\hat{t}|$ is given by

$$
\frac{9}{2 \pi} \frac{\alpha_{s}^{2}}{\hat{t}^{2}}=\frac{\pi^{3}}{2} \frac{\bar{\alpha}^{2}}{\hat{t}^{2}}
$$

The factor $1 / k_{\perp 0}^{2}$ follows because $\mathcal{G}$ is defined as a density in $\ln k_{\perp}^{2}$, rather than in $k_{\perp}^{2}$.

\subsection{Soft Chains}

It should be noted that $\sigma_{\mathrm{c}}$ gives the cross section for a chain with at least one link with virtuality larger than $k_{\perp 0}^{2}$. There will also be chains with no perturbative emissions above this cut. (Emissions below the cut have to be added later as final state radiation.) The cross section for these soft chains, i.e. the cross section for exchanging a single (non-perturbative) gluon with virtuality $k_{\perp 0}^{2}$ (see figure $[\mathrm{b}$ ), is obtained by simply convoluting the two input gluon densities:

$$
\sigma_{\mathrm{c} 0}\left(k_{\perp 0}^{2}\right)=\frac{\pi^{3}}{2 k_{\perp 0}^{2}} \int d x_{0+} d x_{0-} g\left(x_{0+}, k_{\perp 0}^{2}\right) g\left(x_{0-}, k_{\perp 0}^{2}\right) \delta\left(x_{0+} x_{0-}-k_{\perp 0}^{2} / s\right) .
$$

As we will see in section 6, if the cutoff is increased, the cross section for the perturbative chains will decrease, but the cross section for the non-perturbative chains will increase since the fitted input gluon density is then more divergent for small $x$. This implies that the total number of chains in $p p$ collisions is insensitive to the cutoff $k_{\perp 0}$, and therefore can be fully determined by data on $F_{2}$, without any additional adjustable free parameter.

The results in eqs. (3.2) and (3.5) give together with the total non-diffractive cross section the average number of chains in one event. This average number is, however, not enough to determine the properties of exclusive final states. The sub-collisions may be correlated so that central collisions have a larger, and peripheral a smaller, number of chains. These correlations will be studied in more detail in section 1 .

\subsection{Final State Bremsstrahlung}

In the LDC model, final state radiation is emitted from the dipoles in the chain in the same way as in the Dipole Cascade Model (DCM) [29, 30] for time-like parton cascades, which is implemented in the ARIADNE program [31]. In these time-like cascades the emissions are, however, limited by the $k_{\perp}$ of the propagators in the initial chain. In figure 3 , this corresponds to the area below the line connecting the $q_{i}$ points.

The parameters in the DCM have been fitted, together with the parameters in the string fragmentation [32] in PYTHIA, to reproduce a wide range of final-state observables at LEP to an extraordinary precision. The main parameters in ARIADNE are $\Lambda_{\mathrm{QCD}}$ and 


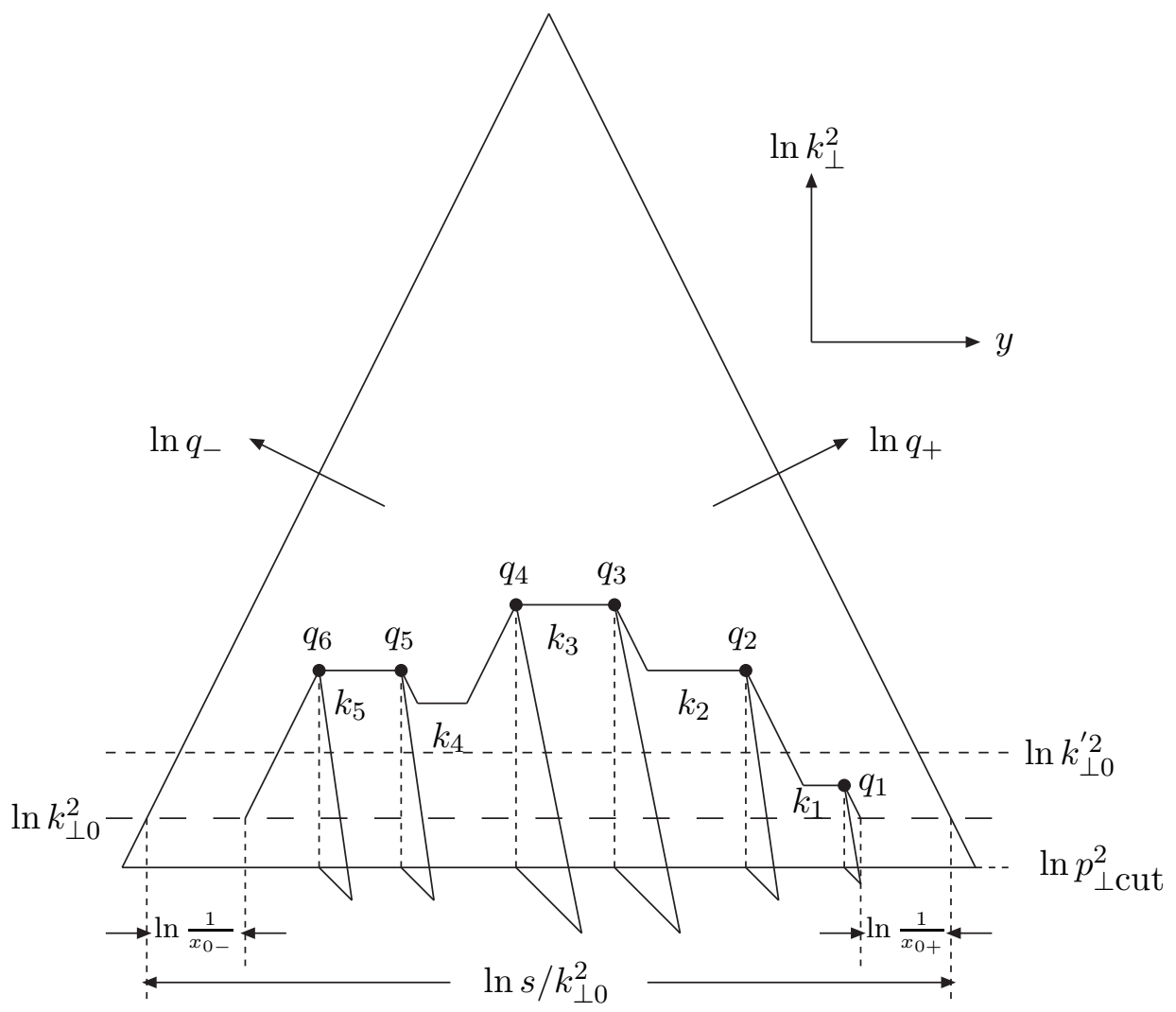

Figure 3: An example of a dipole chain in hadronic collisions. The initial-state emissions $q_{i}$ are marked with points in the $\left(y, \kappa=\ln \left(q_{\perp}^{2}\right)\right)$-plane. The connecting lines correspond to the propagators $k_{i}$.

the cutoff in transverse momentum, $p_{\perp \text { cut }}$, which have been fitted by the DELPHI collaboration to $0.22 \mathrm{GeV}$ and $0.6 \mathrm{GeV}$ respectively [33]. The fact that $p_{\perp \text { cut }}$ typically is smaller than the cutoff for the ISB, $k_{\perp 0}$, obtained by fitting LDC to $F_{2}$ data, means that FSB will be allowed also below $k_{\perp 0}$ down to the hadronization cutoff, $p_{\perp \text { cut }}$. This means that if $k_{\perp 0}$ is increased to $k_{\perp 0}^{\prime}$, some gluon emissions in the initial state, such as $q_{1}$ in figure 3 , will no longer be allowed, but will then instead be allowed as FSB. In this way the distribution of the final state partons should be quite insensitive to the precise value of $k_{\perp 0}$.

Special care must be taken when radiating from the dipoles connecting the first ISB gluons with the hadron remnants. This problem is especially important for the soft chains, where FSB will also be allowed below $k_{\perp 0}$ from a dipole between the remnants. This will be discussed further in section 5 .

\section{Multiple scatterings in hadronic collisions}

At high energies the inclusive cross section for parton-parton sub-collisions becomes larger than the total non-diffractive cross section, which means that there are on average more than one hard collision per event. Indications for multiple hard collisions are observed both in hadronic collisions [10] and in photoproduction [34]. The properties of exclusive final states then depend sensitively on the distribution in the number of sub-collisions in a single 
event. At high energies two colliding hadrons are Lorentz contracted to two flat pancakes. When they pass through each other the different regions are causally disconnected, and it is then natural to assume that the sub-collisions are independent for a fixed value of the impact parameter, $b$. This implies an eikonalized description, where the probability to have $n$ sub-collisions is given by a Poissonian distribution with an average, $\bar{n}(b)$, which depends on $b$, or alternatively by such a distribution with the value $n=0$ excluded. Models of this type include PythiA, HeRWig ${ }^{1}$, and the Dual Parton Model.

\subsection{Impact-Parameter Dependence}

We note that the result depends not only upon the average number of hard sub-collisions for fixed impact parameter, $\tilde{n}(b)$, but also on the probability for an interaction, $P_{\text {int }}(b)$, for this value of $b$. These quantities are defined so that the inclusive sub-collision cross section is given by $\sigma_{\text {hard }}=\int d^{2} b \tilde{n}(b)$ and the total non-diffractive cross section by the relation $\sigma_{\text {tot }}=\int d^{2} b P_{\text {int }}(b)$. Expressed in these quantities we have

$$
\bar{n}(b)=\tilde{n}(b) / P_{\text {int }}(b)
$$

In ref. 10] it is found that e.g. the "pedestal effect", i.e. the fact that the background is larger around a high $E_{\perp}$ jet than in a minimum bias event, and the distribution in the number of jets in a single event can be well reproduced if the $b$-dependence is described by a double Gaussian distribution, corresponding to the presence of a kind of "hard core", while a single Gaussian would severely underestimate the fluctuations. (By a Gaussian distribution we understand here a distribution which is Gaussian in the variable $b$, and consequently exponential in the density $b^{2}$.)

In this paper we will not present a full investigation of such correlations between subcollisions, but only study some qualitative features. The fact that there often are more than two jets in one single chain in our approach, will imply a positive correlation between the sub-collisions in addition to the one originating from the impact parameter dependence. Therefore the fluctuations in the number of chains due to the impact parameter dependence should be weaker than the fluctuations in the number of sub-collisions in the models mentioned above. To illustrate the effect of these two contributions to the correlations, we will study two different cases:

- Completely independent chains, where the number of chains is determined by a Poissonian distribution

$$
P_{n}=e^{-\bar{n}} \frac{\bar{n}^{n}}{n !}
$$

where $\bar{n}$ is the $b$-independent average number of chains in an event.

- An impact parameter dependence which results in an exponential distribution in $\bar{n}(b)$ when weighted by the probability $P_{\text {int }}(b)$. The probability $P\left(n^{\prime}\right)$ for a collision with a centrality corresponding to $\bar{n}(b)=n^{\prime}$ is thus assumed to satisfy

$$
P\left(n^{\prime}\right)=\frac{1}{\sigma_{\text {tot }}} \int d^{2} b P_{\text {int }}(b) \delta\left(\bar{n}(b)-n^{\prime}\right)=\bar{n} e^{-n^{\prime} / \bar{n}}
$$

\footnotetext{
${ }^{1}$ Using the add-on JimMY program [3]]
} 


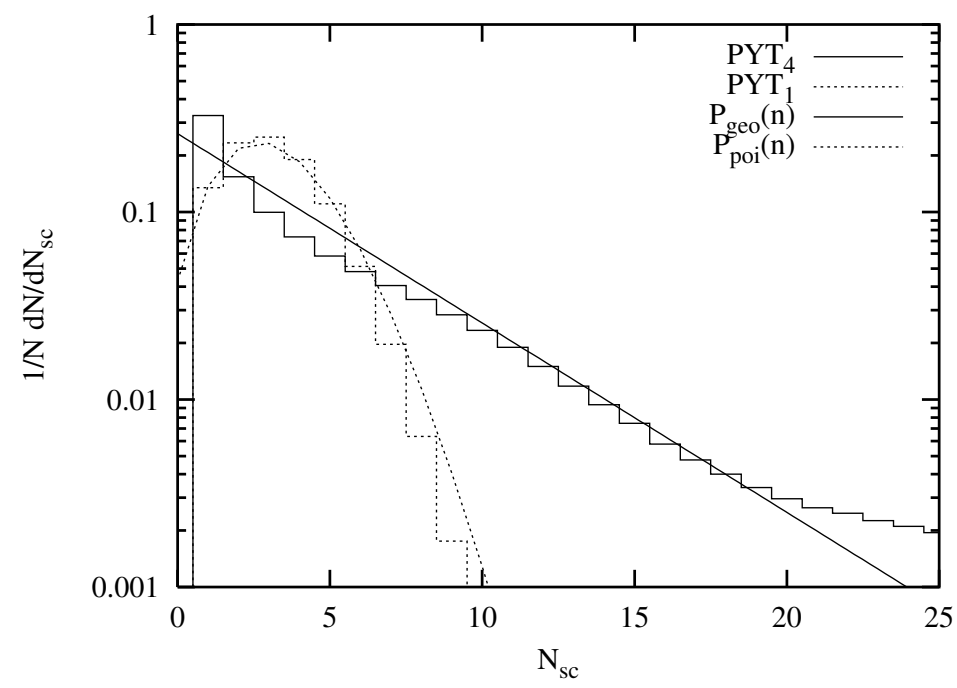

Figure 4: The distribution in number of scatterings for non-diffractive events in PYTHIA. The full histogram is $\mathbf{P} \mathbf{Y} \mathbf{T}_{\mathbf{4}}$ and the dotted histogram is $\mathbf{P Y} \mathbf{T}_{\mathbf{1}}$ (see subsection 6.1 for notation). The full and dotted curves are geometrical and Poissonian distributions respectively with the same average as the corresponding histograms.

Here $\bar{n}$ is the average $n^{\prime}$-value. Assuming a Poissonian distribution in $n$ for fixed value of $b$, i.e. for fixed value of $n^{\prime}$, we then find a geometric distribution with the same average, $\bar{n}$, for the number of chains, $n$, in a random event. For the distribution in the number of chains we then find the geometric distribution in $n$, with the same average, $\bar{n}$ :

$$
P_{n}=\int d n^{\prime} P\left(n^{\prime}\right) \cdot e^{-n^{\prime}} \frac{n^{\prime n}}{n !}=\frac{1}{1+\bar{n}}\left(\frac{\bar{n}}{1+\bar{n}}\right)^{n}
$$

In figure 6 we compare the simple geometrical distribution with the distribution in number of scatterings in PYTHIA with a double Gaussian $b$-distribution, and we find that the geometrical distribution actually is extremely similar. Also shown is PYTHIA with independent scatterings and a Poissonian distribution for comparison.

A reason to concentrate on these two alternatives in this preliminary study, is that the Poissonian distribution will act as a reference for the effect of further correlations, while the geometric distribution can mimic the fluctuations in the favoured PYTHIA version. We also note that both these distributions are chosen so that they depend only on a single parameter, the average number of chains, $\bar{n}$.

\section{Monte Carlo implementation}

The LDC event generator LDCMC, is written to model deeply inelastic lepton-hadron scattering or, more specifically, $\gamma^{*}-h$ scattering. To use it for generating single dipole chains in hadronic collisions we lower the virtuality of the $\gamma^{*}$ to below $k_{\perp 0}$ and use a reweighting procedure to obtain the correct input parton densities for the hadron and to account for the fact that the photon only couples to quarks, while the hadron also has a gluon density. 
Since we expect that there will be several chains in each hadronic collision, we need to discuss two problems concerning how to combine the generated chains: we need to consider the colour connections between the chains and the hadron remnants, and we need to ensure energy-momentum conservation, so that the chains do not take more than the available energy in the colliding hadrons.

\subsection{Colour Connections}

We do not expect all individual chains to be connected to the hadron remnant - this would give a large set of overlapping strings producing too many hadrons in the full rapidity region. Instead we expect that ISB partons in the ends of each chain may be connected to corresponding partons in other chains. One could also imagine situations where two chains are different branches on the same cascading tree. This possibility will, however, be neglected in the present analysis. To be less sensitive to the colour connections we will here only consider the properties of the partonic final state. However, although less sensitive, also the final state radiation will be affected to some extent, since the emitting dipoles depend on these connections.

Clearly, the question of colour connections is a very complicated one, and we will defer a detailed investigation to a later publication. Here we will simply assume that the FSB can be added to each chain independently, also allowing the dipoles between the ISB partons in the ends of the chain and the hadron remnants to radiate. This will certainly somewhat overestimate the amount of soft partons in the events and the results presented below must therefore be considered somewhat preliminary.

The FSB emissions from the remnant dipoles also pose another problem. The emission probability from a dipole is basically flat in $\ln p_{\perp}^{2}$ and rapidity. This means that the emitted gluon can easily take a large fraction of the remnant momentum. The standard way of dealing with this in ARIADNE is the Soft Radiation Model [35], but here we will use a different method, to be as insensitive as possible to the cutoff, $k_{\perp 0}$. Looking at the initial-state emission of the gluon $q_{1}$ in figure 3, it will also have a basically flat rapidity distribution, except there will be a $\left(1-x_{0+}\right)^{4}$ suppression from the input gluon density (see eq. (6.1) and table 1 in section 6.1). If we now increase the cutoff to $k_{\perp 0}^{\prime}, q_{1}$ should instead be allowed as a final state emission with approximately the same distribution. We have therefore chosen a procedure where for each gluon emission generated according to the Dipole Cascade Model in a remnant dipole, a corresponding new $x_{0+}^{\prime}$ is calculated from the energy loss of the remnant, and the emission is only accepted with a probability

$$
\left(\frac{1-x_{0+}^{\prime}}{1-x_{0+}}\right)^{4} \text {. }
$$

The correct procedure would, of course, be to use the actual ratio of the relevant input parton densities. We have, however, checked that the results are insensitive to the details of the suppression (e.g. changing the exponent from 4 to 3 ) and will therefore in this paper be satisfied with this simplified treatment. 


\subsection{Energy-Momentum Conservation}

The treatment of emissions from the remnant dipole is also related to the question of energy-momentum conservation when combining several chains. If each chain takes a large fraction of the energy of the incoming hadron, there will not be enough energy to have many chains. To ensure energy-momentum conservation we have adopted the following procedure when combining chains into single events.

1. First we choose $n$, the number of chains to be included in the event, from either the Poissonian distribution in eq. (4.2) or the geometrical one in eq. (4.4).

2. Then we choose $n$ chains, either hard chains with both ISB and FSB partons or soft ones with only FSB, according to the values of $\sigma_{c}$ and $\sigma_{c 0}$.

3. The selected chains are then ordered in the transverse momentum of the hardest parton. This procedure is analogous to the treatment in the PYTHIA model. It implies that the hardest chain is always accepted in the following veto procedure, which is necessary to ensure that the spectrum of high $E_{\perp}$ jets is correctly described.

4. Each chain is now tested in order of decreasing hardness. The sum of the positive and negative light-cone momentum already used up by the previously accepted chains is calculated and from that, new effective values of $x_{0 \pm}^{\prime}$ of the current chain are obtained. Chains which would take more than the available energy are vetoed. Kinematically allowed chains are accepted with a probability

$$
\left(\frac{1-x_{0+}^{\prime}}{1-x_{0+}} \times \frac{1-x_{0-}^{\prime}}{1-x_{0-}}\right)^{4},
$$

to approximate the change in the input parton densities after some partons have already been extracted. As for the treatment of remnant dipoles in eq. (5.1) it may be more correct to here use the ratio of the actual input densities used. (Again we have checked that our results are not very sensitive if the exponent is changed from 4 to 3 .)

5. We are now left with $n_{\text {acc }} \leq n$ accepted chains which are combined into a partonic event.

The procedure presented here is at present rather cumbersome and slow and some results presented here have rather large statistical fluctuations. The absence of hadronization also makes the current version of the program unsuited for direct comparison with experiment. A new publicly available version of LDCMC capable of generating hadronic collision events will therefore be postponed until the issues of colour connections have been addressed in more detail.

\section{Results}

In this section we will study features of jet and minijet production, which are sensitive to the properties of multiple interactions. We will look at inclusive jet production, minijet distributions, and the pedestal effect and other properties of underlying events. 


\begin{tabular}{|l||r|r|r||r|r||r|r||r|r||r||r|}
\hline fit & $A_{g}$ & $a_{g}$ & $b_{g}$ & $a_{d}$ & $b_{d}$ & $a_{u}$ & $b_{u}$ & $a_{s}$ & $b_{s}$ & $k_{\perp 0}$ & $\chi^{2} /$ d.o.f. \\
\hline fit-0 & 2.24 & 0.09 & $\mathbf{4}$ & 1.77 & $\mathbf{3}$ & 0.58 & $\mathbf{3}$ & $\mathbf{0}$ & $\mathbf{4}$ & $\mathbf{0 . 8 8}$ & $795 / 625$ \\
fit-1 & 1.86 & 0.00 & $\mathbf{4}$ & 1.78 & $\mathbf{3}$ & 0.57 & $\mathbf{3}$ & $\mathbf{0}$ & $\mathbf{4}$ & 0.99 & $694 / 625$ \\
fit-2 & 1.42 & -0.09 & $\mathbf{4}$ & 1.94 & $\mathbf{3}$ & 0.56 & $\mathbf{3}$ & $\mathbf{0}$ & $\mathbf{4}$ & $\mathbf{1 . 1 4}$ & $710 / 625$ \\
fit-3 & 1.17 & -0.14 & $\mathbf{4}$ & 2.10 & $\mathbf{3}$ & 0.55 & $\mathbf{3}$ & $\mathbf{0}$ & $\mathbf{4}$ & $\mathbf{1 . 3}$ & $751 / 625$ \\
\hline
\end{tabular}

Table 1: The result of the fit of the parameters for the input parton densities to data from H1 [36], ZEUS [37], NMC [38] and E665 [39] in the region $x<0.3, Q^{2}>1.5 \mathrm{GeV}^{2}$. The last columns gives the $\chi^{2}$ over the number of fitted data points, respectively. Parameters in bold face have not been fitted.

As example we have chosen $p p$ collisions at the Tevatron energy, $1.8 \mathrm{TeV}$ in the cms. To be specific we define jets with a simple cone-algorithm ${ }^{2}$ using an idealized calorimeter with cells distributed uniformly in pseudo-rapidity and azimuth angle with the size $\delta \eta \times \delta \phi \approx$ $0.2 \times 0.2$. The transverse energy of all generated partons are collected in the corresponding cells, and any cell with a summed $E_{\perp}$ above $1 \mathrm{GeV}$ is tried as a jet-initiator. If the summed $E_{\perp}$ within a radius of 0.7 in the $\eta$ - $\phi$ plane around such an initiator is above $3 \mathrm{GeV}$, this $E_{\perp}$ is assigned to a jet in the $E_{\perp}$ weighted direction of the cells included. Only jets with $|\eta|<2.5$ are included in the following.

As discussed above we will in this preliminary study not include hadronization effects, which are sensitive to how the chains are colour connected. We will therefore only compare with parton distributions generated by the PYTHIA generator, and make no direct comparisons with experimental data.

\subsection{Fitting procedure}

The parameters used in the MC simulations are fitted to DIS data. To get quantitative results from LDC we must convolute the dipole chains with input parton densities in the proton. These densities are not à priori known, but must be parameterized in some way and fitted to data. The form of these densities are taken to be

$$
x f_{i}\left(x, k_{\perp 0}^{2}\right)=A_{i} x^{a_{i}}(1-x)^{b_{i}}
$$

where $i=d_{v}, u_{v}, g$ and $s$ for the d-valence, u-valence, gluon and sea-quark densities respectively (where the sea flavour densities are assumed to be $f_{\bar{d}}=f_{\bar{u}}=2 f_{\bar{s}}$ ). The parameters $A_{i}, a_{i}, b_{i}$ and the perturbative cutoff, $k_{\perp 0}$, are then fitted to reproduce the measured data on $F_{2}$. There are some relations between the parameters which are fixed by sum rules. Thus $A_{d_{v}}$ and $A_{u_{v}}$ are determined by flavour conservation and $A_{s}$ is fixed by momentum conservation. The fits to $F_{2}$ do not constrain the remaining parameters very strongly, so we have fixed the $b$ parameters to 3 in the valence densities and to 4 in the sea and gluon densities. The best fit is the one called standard in reference [28] and fit-1 in table 11. The fitted value of $k_{\perp 0}$ is $0.99 \mathrm{GeV}$, but since we would like to check the dependence on the cutoff, we also present additional fits in table 1 where $k_{\perp 0}$ has been fixed to higher and lower values.

\footnotetext{
${ }^{2}$ PYCELL in PYTHIA
} 
In the following we will look at a couple of options for LDC and compare with different cases in PYTHIA. (For the alternatives with a single chain $\left(\mathbf{L} \mathbf{D} \mathbf{C}_{\mathbf{0}}\right.$ and $\left.\mathbf{L} \mathbf{D} \mathbf{C}_{\mathbf{0}}^{\prime}\right)$ or a single sub-collision $\left(\mathbf{P Y T}_{\mathbf{0}}\right)$ we can only study inclusive cross sections, and the reduction from energy conservation due to overlayed chains or collisions is not included.)

$\mathbf{L D C}_{\mathbf{0}}$ : Single LDC chains with the default (fit-1) tuning.

$\mathbf{L D C}_{\boldsymbol{P}}$ : Uncorrelated multiple LDC chains (fit-1) according to the Poissonian distribution.

$\mathbf{L D C}_{G}$ : Multiple LDC chains (fit-1) according to the geometrical distribution.

$\mathbf{L D C}_{\mathbf{0}}^{\prime}$ : As $\mathbf{L} \mathbf{D C} \mathbf{C}_{\mathbf{0}}$ but with the $k_{\perp 0}=1.3 \mathrm{GeV}$ tuning (fit-3) (similarly for $\mathbf{L} \mathbf{D C}_{\boldsymbol{G}}^{\prime}$ ).

$\mathbf{P Y T}_{\mathbf{0}}$ : PYTHIA without multiple scatterings selecting low- $p_{\perp}$ events without hadronization. Everything else default.

$\mathbf{P Y T}_{\mathbf{1}}$ : As $\mathbf{P} \mathbf{Y} \mathbf{T}_{\mathbf{0}}$ but with uncorrelated multiple scatterings (with default values ${ }^{3}$ ).

$\mathbf{P Y T}_{\mathbf{4}}$ : As $\mathbf{P Y T}_{\mathbf{0}}$ but with multiple scatterings according to a double Gaussian distribution (with default values).

$\mathbf{P Y T}_{4}^{\prime}$ : As $\mathbf{P Y T}_{\mathbf{4}}$ but with a higher soft regularization parameter $(2.5 \mathrm{GeV}$ instead of $2.1 \mathrm{GeV})$.

Since $\mathbf{P Y T}_{\mathbf{4}}$ is known to give a fair description of experimental data, we will in the following use it as a reasonable approximation to nature. We should keep in mind, however, that the results presented here are on parton level only, and that many of the observables studied have not been measured experimentally, so deviations from $\mathbf{P} \mathbf{Y} \mathbf{T}_{\mathbf{4}}$ do not imply that our model is wrong. We will also mostly compare $\mathbf{L D C}_{\boldsymbol{P}}$ with $\mathbf{P} \mathbf{Y} \mathbf{T}_{\mathbf{1}}$, which both are based on the assumption of uncorrelated scatterings; and $\mathbf{L D C} \mathbf{C}_{\boldsymbol{G}}$ with $\mathbf{P} \mathbf{Y} \mathbf{T}_{\mathbf{4}}$, since we have shown in figure 1 that $\mathbf{P} \mathbf{Y} \mathbf{T}_{\mathbf{4}}$ is well approximated by a geometrical distribution. Note, however, that the procedure for energy conservation in LDC severely limits the possibility to have many chains, and $\mathbf{L D C}_{\boldsymbol{G}}$ deviates substantially from a geometrical distribution, as can be seen in figure 5. The effects of energy conservation in PYTHIA is much smaller since the ISB is modeled with a DGLAP parton shower with strongly ordered virtualities which means that only very rarely are partons emitted close to the hadron remnants.

\subsection{Chain multiplicity}

First we want to study the cross section for the formation of a chain in a hadronic collision, which is given by eqs. (3.2) and (3.5). For fit-1 at a c.m. energy of $1.8 \mathrm{TeV}$, we obtain for perturbative chains a cross section of $\sigma_{\mathrm{c}}=315 \mathrm{mb}$, which is much larger than the non-diffractive cross section at the Tevatron ${ }^{4}, \sigma_{\text {nd }} \approx 39 \mathrm{mb}$. In figure 6 we see how this cross section per chain varies with the soft cutoff used in the fit to DIS $F_{2}$ data. The result is an almost linear dependence on the cutoff $k_{\perp 0}$. (Note that the input parton densities have been re-fitted for each value of $k_{\perp 0}$.) In figure 6 we also show the cross section for

\footnotetext{
${ }^{3}$ The indices for the PYTHIA options refer to the setting of the switch, MSTP(82), selecting the multiple interaction model.

${ }^{4}$ We take the value as estimated by a simple parameterization in PYTHIA based on ref. [40].
} 


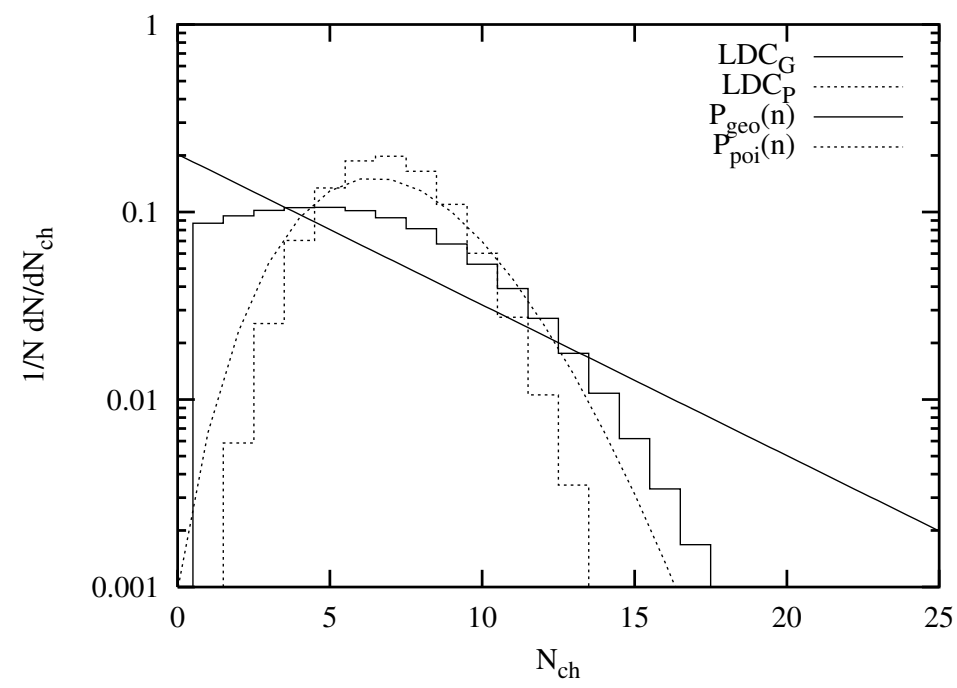

Figure 5: The distribution in number of chains per event in LDC. The full histogram is $\mathbf{L D C}_{\boldsymbol{G}}$ and the dotted histogram is $\mathbf{L} \mathbf{D C} \mathbf{P}_{\boldsymbol{P}}$. The full and dotted curves are geometrical and Poissonian distributions respectively with the same average as the corresponding histograms.

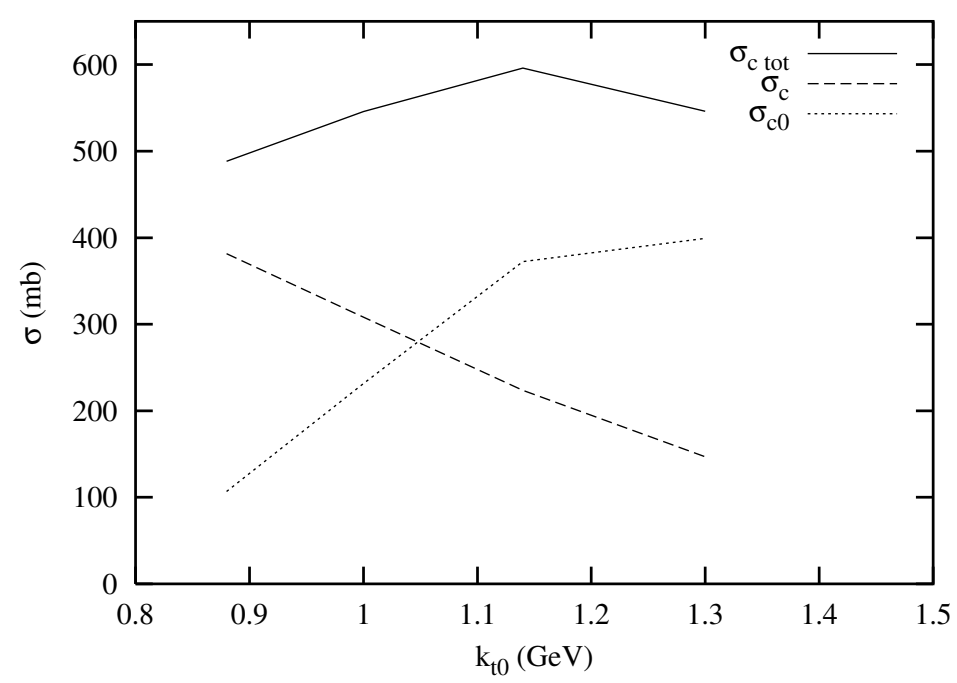

Figure 6: The cross section per chain in the LDC model as a function of the cutoff, $k_{\perp 0}^{2}$. The dashed line is the cross section for chains with at least on emission above the cutoff, the dotted line is for soft chains without emissions above the cutoff, and the full line is the sum of the two. Note that the input parton densities have been re-fitted for each value of $k_{\perp 0}$.

formation of soft chains, represented by the exchange of a single (non-perturbative) gluon with virtuality $k_{\perp 0}^{2}$ (cf. eq. (3.5)). We see that if the cutoff is increased, the cross section for the perturbative chains will decrease, but the cross section for the non-perturbative chains will increase since the fitted input gluon density is then more divergent for small $x$. From figure 6 we see that the sum of the two cross sections is almost independent of the cutoff $k_{\perp 0}$. Thus we conclude that the total number of chains in $p p$ collisions can be fully 
(a)

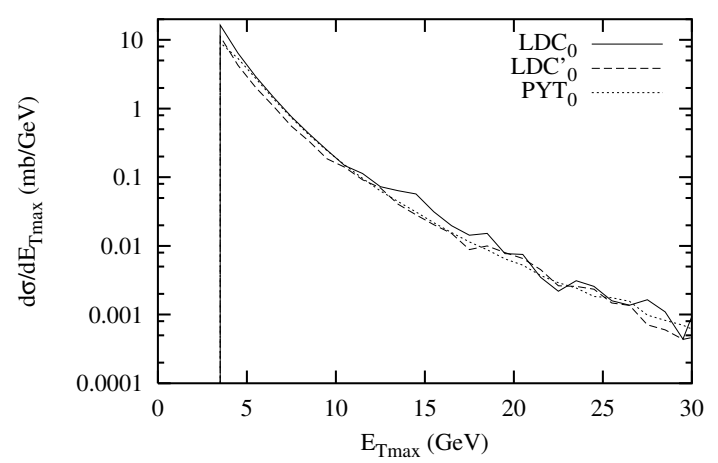

(b)

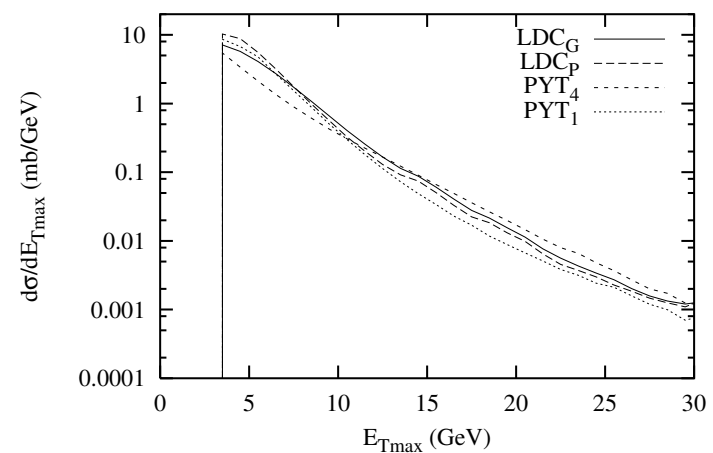

Figure 7: The differential $E_{\perp \max }$ cross section. In (a) $E_{\perp \max }$ is the largest $E_{\perp}$ jet in a chain or hard scattering. Full line is $\mathbf{L} \mathbf{D C}_{\mathbf{0}}$, dashed is $\mathbf{L} \mathbf{D C} \mathbf{0}_{\mathbf{0}}^{\prime}$ and the dotted line is $\mathbf{P} \mathbf{Y} \mathbf{T}_{\mathbf{0}}$. In (b) $E_{\perp \max }$ is the largest $E_{\perp}$ in an event. Full line is $\mathbf{L D C} \mathbf{G}_{\boldsymbol{G}}$, long-dashed is $\mathbf{L} \mathbf{D C} \mathbf{C}_{\boldsymbol{P}}$, short-dashed is $\mathbf{P} \mathbf{Y} \mathbf{T}_{\mathbf{4}}$ and the dotted line is $\mathbf{P Y T}_{\mathbf{1}}$

determined by data on $F_{2}$, without any additional adjustable free parameter.

Taking the sum of $\sigma_{c}$ and $\sigma_{c 0}\left(547 \mathrm{mb}\right.$ for $\left.k_{\perp 0}=0.99 \mathrm{GeV}\right)$ we would then expect on the average around 14 chains per collision. However, after the veto procedure to conserve energy and momentum we are left with an average of 7 and 6 chains per event for $\mathbf{L D C}_{\boldsymbol{P}}$ and $\mathbf{L D C} \mathbf{C}_{\boldsymbol{G}}$ respectively.

\subsection{Inclusive jet production}

In figure $7 \mathrm{a}$ a we look at the inclusive differential cross section for the highest $E_{\perp}$ jet in a single LDC chain and compare it to the corresponding cross section in PYTHIA without multiple interactions $\left(\mathbf{P Y T}_{\mathbf{0}}\right)$. We find that the LDCMC result is close to PYTHIA. Comparing $\mathbf{L} \mathbf{D C _ { 0 }}$ and $\mathbf{L} \mathbf{D C} \mathbf{0}_{\mathbf{0}}$ we find a weak dependence on the cutoff. The reason for this may be that the input densities in LDC has been fitted to $F_{2}$ data which are only indirectly sensitive to the gluon contribution. Indeed, in $\mathbf{L D C}_{\mathbf{0}}^{\prime}$ the gluons carry a smaller fraction of the proton momentum which could account for the differences in figure $7 \mathrm{a}$. In the future, when LDCMC can be compared directly with high- $E_{\perp}$ jet production, it should be possible to make a more global fit of the input parton densities, which would better constrain the gluon distribution. Another reason for the dependence could be the differences in FSB caused by our simplified treatment of the colour connections of the chains.

In figure $7 \mathrm{~b}$ we show the differential $E_{\perp \max }$ cross with multiple interactions added with Poissonian $\left(\mathbf{L D C}_{\boldsymbol{P}}\right)$ and geometrical $\left(\mathbf{L} \mathbf{D C}_{\boldsymbol{G}}\right)$ distributions for the number of chains in one event. For comparison we also show $\mathbf{P} \mathbf{Y} \mathbf{T}_{\mathbf{1}}$ and $\mathbf{P} \mathbf{Y} \mathbf{T}_{\mathbf{4}}$. We find that the LDC curves more or less lie in between the PYTHIA ones, which indicates that the procedure chosen to combine chains is a reasonable one. We note, however, a difference for small $E_{\perp \text { max }}$ values where $\mathbf{L D C} \mathbf{C}_{\boldsymbol{G}}$ lies significantly above $\mathbf{P Y T}_{\boldsymbol{4}}$. 
(a)

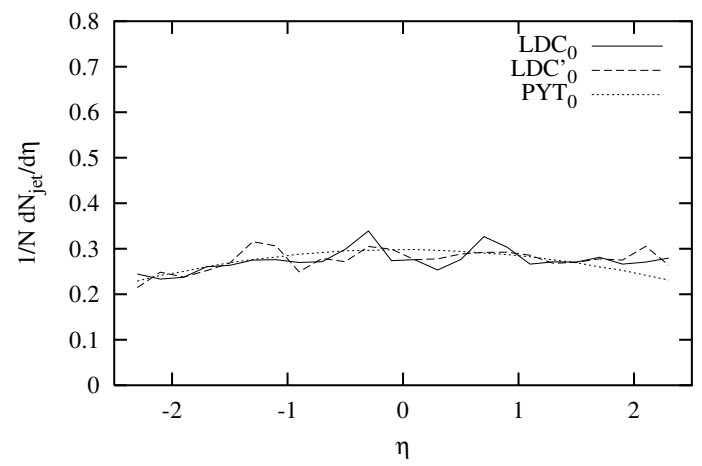

(b)

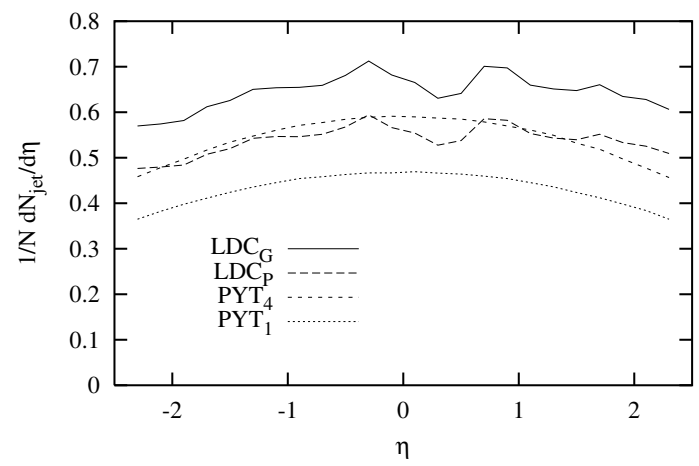

Figure 8: The rapidity distribution of minijets (per event with at least one minijet) In (a) the full line is $\mathbf{L} \mathbf{D C}_{\mathbf{0}}$, dashed is $\mathbf{L} \mathbf{D C} \mathbf{0}_{\mathbf{0}}$ and the dotted line is $\mathbf{P} \mathbf{Y} \mathbf{T}_{\mathbf{0}}$. In (b) the full line is $\mathbf{L} \mathbf{D C} \mathbf{G}_{\boldsymbol{G}}$, long-dashed is $\mathbf{L D C} \mathbf{P}_{\boldsymbol{P}}$, short-dashed is $\mathbf{P Y} \mathbf{T}_{\mathbf{4}}$, dash-dotted is $\mathbf{P Y} \mathbf{T}_{\mathbf{3}}$ and the dotted line is $\mathbf{P} \mathbf{Y} \mathbf{T}_{\mathbf{4}}$. (The different LDC curves have been obtained using the same sample of chains which is why the statistical fluctuations are correlated.)

\subsection{Minijet distributions}

As another check that our procedure is reasonable, we look at the rapidity distribution of minijets. In figure $8 \mathrm{a}$ we show the results for $\mathbf{L D C} \mathbf{C}_{\mathbf{0}}, \mathbf{L} \mathbf{D C} \mathbf{C}_{\mathbf{0}}$ and $\mathbf{P} \mathbf{Y} \mathbf{T}_{\mathbf{0}}$. We find that the curves agree well, and the cutoff dependence in LDC is negligible. The slight asymmetry in the LDC distributions is due to the fact that the recoil strategy for the final state emissions from the parton closest to the photon in the LDCMC for DIS is not fully consistently modified in this preliminary application to hadronic collisions.

In figure $8 \mathrm{~b}$ we show the results for the multiple interaction models. Here the LDC curves are above the corresponding PYTHIA ones. Although there are no obvious reasons for the curves to agree, we note that the soft FSB in LDC may be somewhat overestimated, especially for the soft chains. We also note that the statistics for the LDC curves is poor due to the present inefficient simulation procedure. In addition, the statistical fluctuations in $\mathbf{L D C} \mathbf{C}_{\boldsymbol{P}}$ and $\mathbf{L D C} \mathbf{C}_{\boldsymbol{G}}$ are correlated since the two curves are obtained using the same set of generated single LDC chains.

To investigate the fluctuations we show in figure 9 a the distribution in number of jets in the central 5 units of rapidity for the different multiple interaction models. It is clear that $\mathbf{L D C}_{\boldsymbol{G}}$ has much larger fluctuations than has $\mathbf{L} \mathbf{D} \mathbf{C}_{\boldsymbol{P}}$, as was expected. $\mathbf{L} \mathbf{D} \mathbf{C}_{\boldsymbol{P}}$ has also larger fluctuations than $\mathbf{P Y T}_{\mathbf{1}}$ although both are based on uncorrelated scatterings. This is because in LDC there is, as mentioned above, a possibility to have several hard scatterings in each chain, which increases the correlations. $\mathbf{L D C}_{\boldsymbol{G}}$ does not have as large fluctuations as $\mathbf{P Y} \mathbf{T}_{4}$, which also was expected since, as shown in figure 5, the distribution in number of chains in $\mathbf{L D C} \boldsymbol{G}$ is much narrower than a geometrical distribution, due to energy conservation. 
(a)

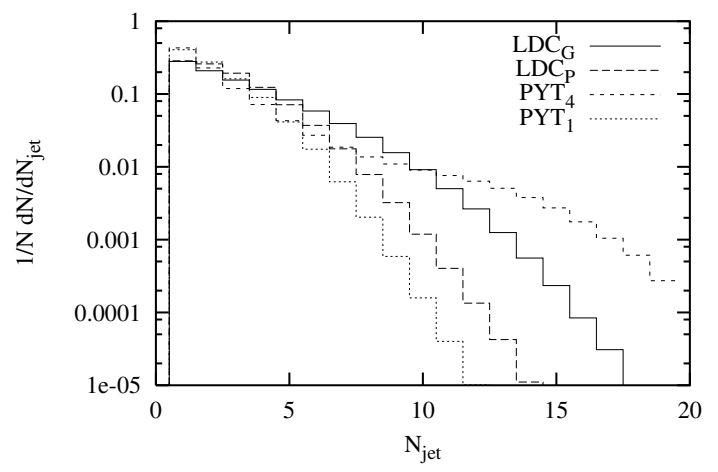

(b)

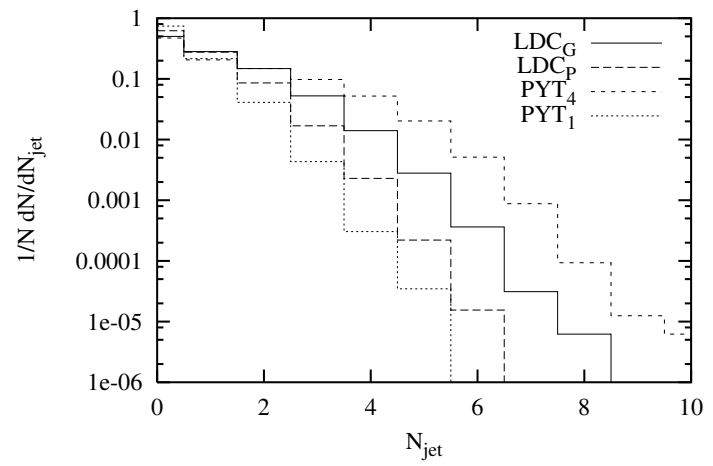

Figure 9: The distribution in number of minijets in the range $|\eta|<2.5$ (per event with at least one minijet). (a) shows all minijets, while (b) is all minijets in the minimum azimuth region (as defined in the text) in each event with $E_{\perp \max }>10 \mathrm{GeV}$. In both cases the full line is $\mathbf{L D C}_{\boldsymbol{G}}$, long-dashed is $\mathbf{L D C} \mathbf{P}_{\boldsymbol{P}}$, short-dashed is $\mathbf{P Y} \mathbf{T}_{\mathbf{4}}$, and the dotted line is $\mathbf{P Y} \mathbf{T}_{\mathbf{1}}$.

\subsection{The Pedestal Effect and Underlying events}

The contribution from hard sub-collisions is in many analyses added on top of an independent "underlying event". In reference [41] a number of observables were presented which were shown to be especially sensitive to this background activity. The regions of azimuth which are transverse to the highest $E_{\perp}$ jet in an event given by $60^{\circ}<\phi<120^{\circ}$, should be less affected by the primary trigger scatterings, which mainly populate the collinear and opposite azimuth region from the highest $E_{\perp}$ jet with hadrons. There are, however, also effects from bremsstrahlung from the primary interaction in the transverse regions, but the authors argue that these typically only affect one of these two regions. Thus, by looking at the activity in the transverse region which has the smallest activity, one is especially sensitive to effects of the "underlying event" and secondary scatterings. We will call this region the minimum azimuth region, and in $9 \mathrm{~b}$ we show the distribution in number of jets in this region in events with a trigger jet of $E_{\perp \max }>10 \mathrm{GeV}$. Clearly, the general trend found for the overall distribution in number of jets is present also here.

One of the most important features of the underlying event is the so-called jet pedestal effect, i.e. the fact that the underlying event activity is not independent of a large $E_{\perp}$ jet. In figure $\sqrt{10}$ we show the average number of minijets as a function of the $E_{\perp}$ of the hardest jet, both for the overall minijet activity and for the minimum azimuth region. As expected all models show an increasing average number with increasing $E_{\perp \max }$ since, even in the case of uncorrelated scatterings, there is a higher probability to find a large $E_{\perp}$ jet in an event with many scatterings than in an event with few. Also, the models with most fluctuations gives the strongest rise, while models with fewer fluctuations saturate earlier. We note, however, that for this variable there is a particularly large difference between the $\mathbf{L D C}_{\boldsymbol{G}}$ and $\mathbf{P Y T}_{4}$ models. 
(a)

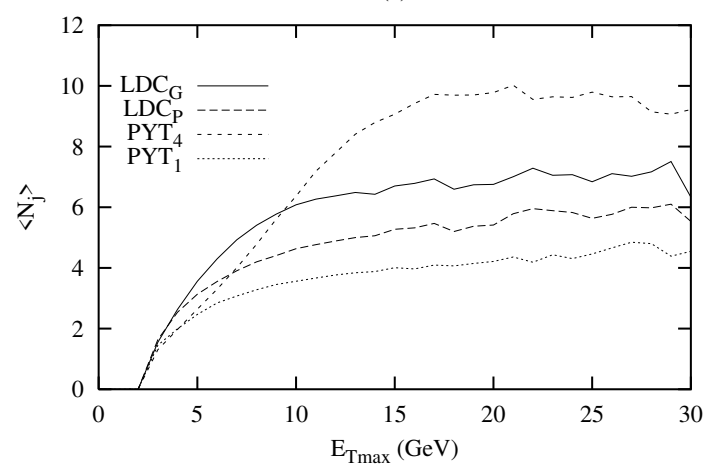

(b)

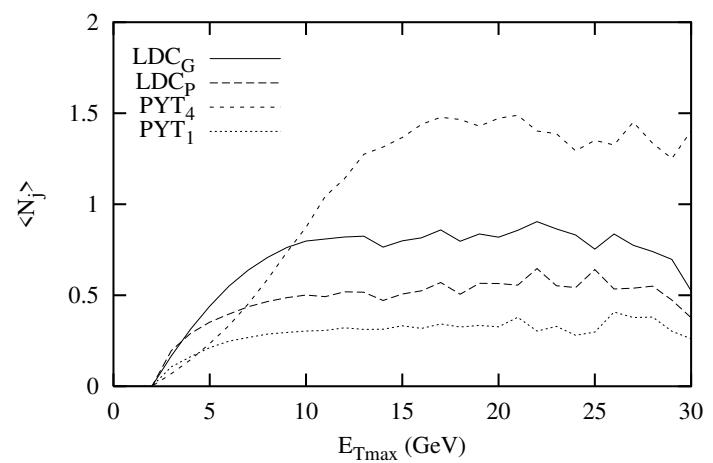

Figure 10: (a) The average number number of minijets per event as a function of the $E_{\perp}$ of the largest jet in the event. The full line is $\mathbf{L D C} \boldsymbol{G}_{\boldsymbol{G}}$, long-dashed is $\mathbf{L} \mathbf{D C} \boldsymbol{P}$, short-dashed is $\mathbf{P Y} \mathbf{T}_{\mathbf{4}}$ and the dotted line is $\mathbf{P Y} \mathbf{T}_{\mathbf{1}}$. (b) Is the same but looking only at minijets in the minimum azimuth region.

(a)

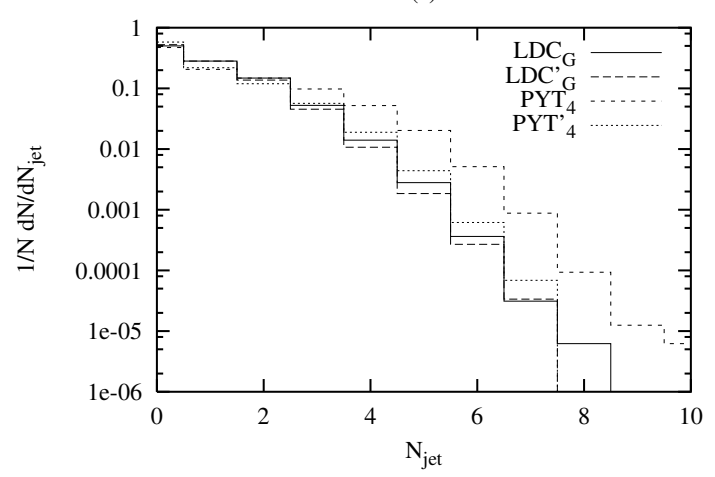

(b)

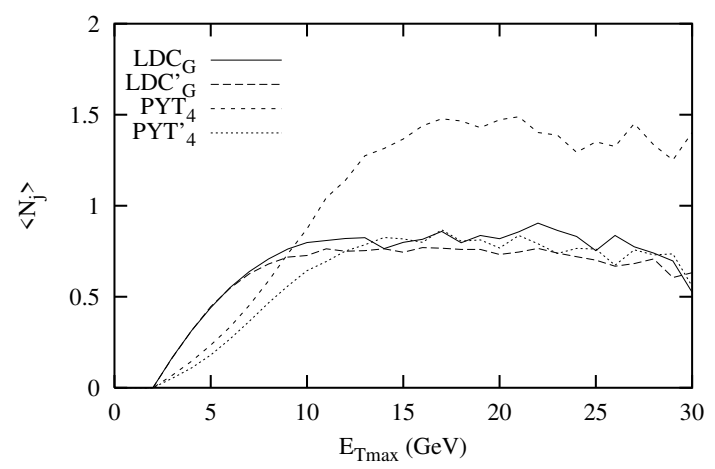

Figure 11: (a) The distribution in number of minijets in the minimum azimuth region in events with $E_{\perp \max }>10 \mathrm{GeV}$. (b) The average number of minijets per event in the minimum azimuth region as a function of the $E_{\perp \max }$. In both plots the full line is $\mathbf{L D C}_{\boldsymbol{G}}$, long-dashed is $\mathbf{L D C} \mathbf{G}_{\boldsymbol{G}}^{\prime}$, short-dashed is $\mathbf{P} \mathbf{Y} \mathbf{T}_{\mathbf{4}}$ and the dotted line is $\mathbf{P} \mathbf{Y} \mathbf{T}_{\mathbf{4}}^{\prime}$.

\subsection{Cutoff Dependence}

Finally we would like to check the cutoff dependence also for our multiple scattering model. In figure 11 we show the distribution in number of jets and the average number of jets as a function of $E_{\perp \max }$ for $\mathbf{L} \mathbf{D C} \mathbf{C}_{\boldsymbol{G}}$ and $\mathbf{L} \mathbf{D C}_{\boldsymbol{G}}^{\prime}$ and we find that the differences indeed are very small. As comparison we also show the results for $\mathbf{P} \mathbf{Y} \mathbf{T}_{\mathbf{4}}$ and $\mathbf{P} \mathbf{Y} \mathbf{T}_{\mathbf{4}}^{\prime}$. For the double-Gaussian model in PYTHIA, there is no direct cutoff, but rather a soft regularization parameter, $p_{\perp 0}$, which forces the partonic cross sections and the running of $\alpha_{s}$ to be finite at small scales by modifying them according to

$$
\frac{d \sigma_{\mathrm{part}}\left(p_{\perp}^{2}\right)}{d p_{\perp}^{2}} \rightarrow \frac{d \sigma_{\mathrm{part}}\left(p_{\perp}^{2}\right)}{d p_{\perp}^{2}} \times\left(\frac{p_{\perp}^{2}}{p_{\perp}^{2}+p_{\perp 0}^{2}}\right)^{2}, \quad \alpha_{s}\left(p_{\perp}^{2}\right) \rightarrow \alpha_{s}\left(p_{\perp}^{2}+p_{\perp 0}^{2}\right)
$$


We see in figure 11 that the result is very sensitive to the value of $p_{\perp 0}$, which has to be adjusted to fit experimental data. We also note that in PYTHIA the value of $p_{\perp 0}$ is expected to increase with energy in a way which is not determined by the model, which means that it is difficult to make reliable predictions for future experiments at higher energies. Naturally we should not compare the variation in cutoff for LDC $(0.99 \rightarrow 1.3 \mathrm{GeV})$ with the variation in the regularization parameter in PүтніA $(2.1 \rightarrow 2.5 \mathrm{GeV})$. From figure 11 we can, however, see that the dependence in LDC is very weak, which means that in this model the result can be directly estimated from data from DIS.

\section{Conclusions}

In this article we present a new approach to describe high energy hadronic collisions, which is based on the Linked Dipole Chain model for DIS. The main advantage over earlier treatments is a much reduced sensitivity to an infrared cutoff, which in models based on collinear factorization has to be fitted to experimental data. In our model the partonparton sub-collision cross section can be fully determined by data from DIS, which implies a more intimate relation between hadron-hadron collisions and DIS. We hope that this relation also implies increased possibilities to use data on $h \mathrm{~h}$ collisions to constrain fits to parton distribution functions. Thus e.g. the connections between different parton chains in $h$ collisions may be related to gluon saturation effects in DIS. The insensitivity to a soft cutoff also makes it easier to make reliable extrapolations to higher energies.

At high energies the properties of hadron-hadron collisions appear to be dominated by hard and semi-hard parton collisions. Although the parton-parton collisions are of perturbative nature, various non-perturbative effects make a description of hadronic collisions much more complicated than e.g. DIS:

(i) In a traditional collinear factorization scheme the inclusive sub-collision cross section is divergent for small $p_{\perp}$, which has to be treated by a soft cutoff $p_{\perp 0}$.

(ii) The inclusive sub-collision cross section is larger than the total non-diffractive cross section, which means that there may be many hard sub-collisions in a single event. This implies that the correlations between the sub-collisions are important.

(iii) The scattered partons can be colour connected with each other and with the proton remnants in different ways by strings or cluster chains.

In models based on collinear factorization the soft cutoff, $p_{\perp 0}$, has to be fitted to experimental data. The correlation between partonic sub-collisions is, in e.g. the DPM model, PYTHIA, and HERWIG, described in an eikonalized form by an impact parameter dependence. For a single hard sub-collision the colour structure can be read off from the perturbative matrix elements [42, 43], but for events with many sub-collisions the result necessarily depends on non-perturbative effects.

The LDC model for DIS is a reformulation and generalization of the CCFM model. It is based on $k_{\perp}$-factorization and the inclusion of non- $k_{\perp}$-ordered parton chains, which 
are expected to be important for limited $p_{\perp}$ and high energies. The formulation is fully symmetric with respect to the photon and the proton ends of the parton chain, which makes the model also suitable for a description of hadronic collisions. The input soft gluon distribution, $g_{0}\left(x, Q_{0}^{2}\right)$, is fitted to data from DIS, and when we then calculate the number of parton chains in $p p$ collisions, we find that the result is actually fixed by the fit to $F_{2}$. If the soft cutoff used in the fit is increased the number of hard chains is reduced, but this is fully compensated by an increase of soft chains described by the input distribution function, $g_{0}$. (These soft chains are now also allowed to emit final state radiation up to the increased value for the $k_{\perp}$-cutoff.)

Concerning point (ii) in the list above, we note that some correlations between hard sub-collisions are included automatically, as a single non- $k_{\perp}$-ordered parton chain can contain two or more local maxima which all correspond to separate hard sub-collisions. Besides this correlation due to sub-collisions in a single chain, we expect that the number of chains is larger in central and smaller in peripheral collisions. As in the models mentioned above, we want to describe this by an impact parameter dependence, and in this paper we have studied two different distributions, one purely stochastic and one similar to the double Gaussian distribution used in the PYTHIA model.

The third problem, concerning colour connections, is very important for the final distribution of hadrons, but less important for a determination of the partonic state before its hadronization. In this preliminary study of the model we focus on features of jet and minijet production, such as the inclusive jet production, minijet distributions and the jet pedestal effect, which are less sensitive to hadronization effects. We will postpone a more detailed analysis of the colour connection structures to future work. In this study we therefore do not include any hadronization effects, and as a consequence we are not comparing our results with experimental data, but are satisfied by a comparison with parton level results from the PYTHIA generator.

For the numerical estimates we are using a modified version of the LDCMC event generator program, and as an example we study $p p$ collisions at $\sqrt{s}=1.8 \mathrm{TeV}$. From the numerical results we realize that it is very essential to consider the effects of energy-momentum conservation. This reduces the possibility to have many chains in a single event, and it also constrains the final state radiation in the regions close to the proton remnants.

On a qualitative level the results from our two versions agree well with the ones obtained from PYTHIA. We find that our version with uncorrelated chains gives larger fluctuations than the corresponding model with uncorrelated scatterings in PYTHIA, which is expected since the dipole chains already include some correlations between multiple scatterings in the same chain. Including the correlations from our geometrical chain distribution increases the fluctuations, but not fully to the level of the model based on a double Gaussian impact parameter dependence in PYTHIA. This is because the suppression of large chainmultiplicities due to the energy conservation procedure, is larger than the corresponding suppression in PYTHIA.

As discussed above, there are still unsolved problems in the LDC model for hadronic collisions. This concerns in particular the modeling of colour connections between the chains and the hadron remnants in a single event, which needs to be analysed in much 
more detail. In turn, this will affect the way final state bremsstrahlung and hadronization are handled, and also the impact of energy conservation. In particular, the simplified model presented here probably overestimates the soft final state emissions, since the final state radiation is allowed from each chain independently. With proper treatment of colour connections we expect a reduction of such soft emissions.

Furthermore, the implementation of the model in the LDCMC event generator needs to be improved substantially, as the current procedure, where $\gamma^{*}$-p collision events need to be reweighted and combined by hand into p-p events, is clearly too cumbersome.

Despite the shortcomings of the simplified model presented here, we are confident that the problems can be solved and that the final model will be able to describe essential features of high energy hadronic collisions, including underlying events and jet-pedestal effects in high $E_{\perp}$ events.

\section{Acknowledgments}

We would like to thank Torbjörn Sjöstrand for valuable discussions.

\section{References}

[1] P. Huovinen, P. V. Ruuskanen, and J. Sollfrank Nucl. Phys. A650 (1999) 227-244, nucl-th/9807076.

[2] B. Andersson, G. Gustafson, and B. Nilsson-Almqvist Nucl. Phys. B281 (1987) 289.

[3] B. Andersson, G. Gustafson, and H. Pi Z. Phys. C57 (1993) 485-494.

[4] A. Capella and J. Tran Thanh Van Z. Phys. C10 (1981) 249-262.

[5] A. Capella, U. Sukhatme, C.-I. Tan, and J. Tran Thanh Van Phys. Rept. 236 (1994) 225-329.

[6] P. Aurenche et al. Phys. Rev. D45 (1992) 92-105.

[7] F. E. Paige, S. D. Protopopescu, H. Baer, and X. Tata hep-ph/9804321.

[8] G. Corcella et al. JHEP 01 (2001) 010, hep-ph/0011363.

[9] T. Sjöstrand, et al. Comput. Phys. Commun. 135 (2001) 238-259, arXiv:hep-ph/0010017.

[10] T. Sjostrand and M. van Zijl Phys. Rev. D36 (1987) 2019.

[11] V. N. Gribov and L. N. Lipatov Yad. Fiz. 15 (1972) 781-807.

[12] L. N. Lipatov Sov. J. Nucl. Phys. 20 (1975) 94-102.

[13] G. Altarelli and G. Parisi Nucl. Phys. B126 (1977) 298.

[14] Y. L. Dokshitzer Sov. Phys. JETP 46 (1977) 641-653.

[15] E. A. Kuraev, L. N. Lipatov, and V. S. Fadin Sov. Phys. JETP 45 (1977) 199-204.

[16] I. I. Balitsky and L. N. Lipatov Sov. J. Nucl. Phys. 28 (1978) 822-829.

[17] G. Gustafson and G. Miu Phys. Rev. D63 (2001) 034004, hep-ph/0002278.

[18] B. Andersson, G. Gustafson, and J. Samuelsson Nucl. Phys. B467 (1996) 443-478.

[19] B. Andersson, G. Gustafson, and H. Kharraziha Phys. Rev. D57 (1998) 5543-5554, hep-ph/9711403.

[20] H. Kharraziha and L. Lönnblad JHEP 03 (1998) 006, hep-ph/9709424.

[21] V. S. Fadin and L. N. Lipatov Phys. Lett. B429 (1998) 127-134, hep-ph/9802290. 
[22] M. Ciafaloni Nucl. Phys. B296 (1988) 49.

[23] S. Catani, F. Fiorani, and G. Marchesini Phys. Lett. B234 (1990) 339.

[24] H. Jung Comput. Phys. Commun. 143 (2002) 100-111, hep-ph/0109102.

[25] Small x Collaboration, B. Andersson et al. hep-ph/0204115.

[26] CTEQ Collaboration, H. L. Lai et al. Eur. Phys. J. C12 (2000) 375-392, hep-ph/9903282.

[27] A. D. Martin, R. G. Roberts, W. J. Stirling, and R. S. Thorne Eur. Phys. J. C23 (2002) 73-87, hep-ph/0110215.

[28] G. Gustafson, L. Lönnblad, and G. Miu JHEP 09 (2002) 005, hep-ph/0206195.

[29] G. Gustafson Phys. Lett. B175 (1986) 453.

[30] G. Gustafson and U. Pettersson Nucl. Phys. B306 (1988) 746.

[31] L. Lönnblad Comput. Phys. Commun. 71 (1992) 15-31.

[32] B. Andersson, G. Gustafson, G. Ingelman, and T. Sjostrand Phys. Rept. 97 (1983) 31.

[33] K. Hamacher and M. Weierstall, "The Next Round of Hadronic Generator Tuning Heavily Based on Identified Particle Data," hep-ex/9511011.

[34] J. M. Butterworth, J. R. Forshaw, and M. H. Seymour Z. Phys. C72 (1996) 637-646, hep-ph/9601371.

[35] Andersson, Bo and Gustafson, Gosta and Lönnblad, Leif and Pettersson, Ulf Z. Phys. C43 (1989) 625.

[36] H1 Collaboration, S. Aid et al. Nucl. Phys. B470 (1996) 3-40, hep-ex/9603004.

[37] ZEUS Collaboration, M. Derrick et al. Z. Phys. C72 (1996) 399-424, hep-ex/9607002.

[38] New Muon Collaboration, M. Arneodo et al. Phys. Lett. B364 (1995) 107-115, hep-ph/9509406.

[39] E665 Collaboration, M. R. Adams et al. Phys. Rev. D54 (1996) 3006-3056.

[40] A. Donnachie and P. V. Landshoff Phys. Lett. B296 (1992) 227-232, hep-ph/9209205.

[41] CDF Collaboration, T. Affolder et al. Phys. Rev. D65 (2002) 092002.

[42] G. Gustafson Z. Phys. C15 (1982) 155-160.

[43] H. U. Bengtsson Comput. Phys. Commun. 31 (1984) 323. 